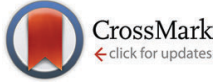

Cite this: Phys. Chem. Chem. Phys., 2016, 18, 25474

Received 15th July 2016, Accepted 19th August 2016

DOI: $10.1039 / c 6 c p 04941 a$

www.rsc.org/pccp

\title{
The impact of environment and resonance effects on the site of protonation of aminobenzoic acid derivatives $\dagger$
}

\author{
Jongcheol Seo, $\ddagger^{a}$ Stephan Warnke, $\ddagger^{a}$ Sandy Gewinner, ${ }^{a}$ Wieland Schöllkopf, ${ }^{a}$ \\ Michael T. Bowers, ${ }^{b}$ Kevin Page ${ }^{* a c}$ and Gert von Helden ${ }^{* a}$
}

\begin{abstract}
The charge distribution in a molecule is crucial in determining its physical and chemical properties. Aminobenzoic acid derivatives are biologically active small molecules, which have two possible protonation sites: the amine ( $\mathrm{N}$-protonation) and the carbonyl oxygen ( $\mathrm{O}$-protonation). Here, we employ gas-phase infrared spectroscopy in combination with ion mobility-mass spectrometry and density functional theory calculations to unambiguously determine the preferred protonation sites of $p-, m-$, and $\mathrm{o}$-isomers of aminobenzoic acids as well as their ethyl esters. The results show that the site of protonation does not only depend on the intrinsic molecular properties such as resonance effects, but also critically on the environment of the molecules. In an aqueous environment, $N$-protonation is expected to be lowest in energy for all species investigated here. In the gas phase, $O$-protonation can be preferred, and in those cases, both $\mathrm{N}$ - and $\mathrm{O}$-protonated species are observed. To shed light on a possible proton migration pathway, the protonated molecule-solvent complex as well as proton-bound dimers are investigated.
\end{abstract}

\section{Introduction}

The properties and reaction dynamics of ionic molecules are crucially determined by the location of the charge..$^{1-7}$ The existence of so-called protomers ${ }^{8,9}$ - molecular isomers that only differ in the site of protonation - is therefore of great importance for both fundamental research and applications. In this context protomers of small biologically active molecules, such as metabolites, hormones, and pharmaceutical substances, are of great interest. These molecules perform their particular function in highly diverse biological environments in which the site of protonation might change significantly. A priori knowledge of the tendency to form protomers is, therefore, not only crucial for a better understanding of these molecules in biological processes but can also help to specifically modulate their function.

\footnotetext{
${ }^{a}$ Fritz-Haber-Institut der Max-Planck-Gesellschaft, Faradayweg 4-6, 14195 Berlin, Germany.E-mail: helden@fhi-berlin.mpg.de

${ }^{b}$ Department of Chemistry and Biochemistry, University of California Santa Barbara, Santa Barbara, California 93106, USA

${ }^{c}$ Freie Universität Berlin, Department of Biology, Chemistry and Pharmacy, Takustrasse 3, 14195 Berlin, Germany. E-mail: kevin.pagel@fu-berlin.de

$\dagger$ Electronic supplementary information (ESI) available: Time-of-flight mass spectra of samples, optimized geometries of molecular ions. See DOI: 10.1039/ c6ср04941a

\$ These authors contributed equally to this work.
}

Aminobenzoic acids (ABA) and their corresponding ethyl esters (ABE) are prototypical examples of molecules that offer two possible protonation sites: their amine and carbonyl groups (Fig. 1). These molecules can be biologically active and are often used as building blocks for drugs or other biologically active molecules with applications ranging from antibacterial substances to UV protective agents. ${ }^{10-13} \mathrm{~A}$ widely known molecule in that context is $p$-ABE, also known as benzocaine, which is a common local anesthetic. ${ }^{14}$ Further, ABA and ABE derivatives often occur as metabolites with, for example, $p$-ABA being a precursor for folic acid ${ }^{15}$ and coenzyme $\mathrm{Q},{ }^{16}$ and $o$-ABA being involved in the biosynthesis of tryptophan in microorganisms. ${ }^{17}$

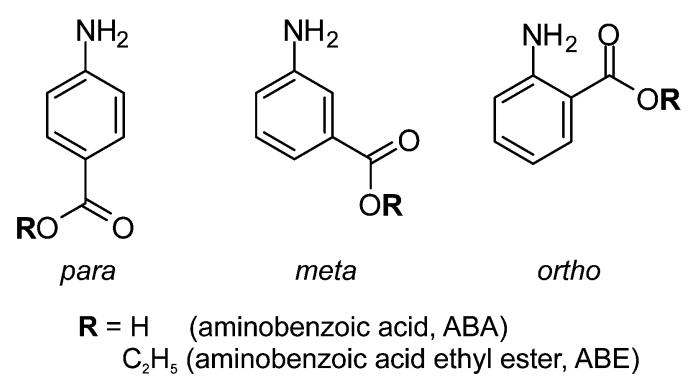

Fig. 1 Chemical structures of $p-, m-$, and $o$-aminobenzoic acids (ABAs, $\mathbf{R}=\mathrm{H}$ ) and their ethyl esters (ABEs, $\mathbf{R}=\mathrm{CH}_{2} \mathrm{CH}_{3}$ ). 
From a chemical point of view, ABA derivatives are of fundamental interest, as different relative positions of the functional groups at the aromatic ring (para, meta, and ortho) can yield significant differences in chemical properties. Three main effects play a role: (a) the inductive effect in which the charge distribution is influenced by the electronegativity of the substituents; (b) the resonance (or mesomeric) effect, caused by p-orbital overlap and (c) the interaction of the molecule and its charge distribution with the environment.

Due to their relative electronegativities, the amine group is electron-donating while the carboxylic acid (or ester) is an electron-withdrawing group. In addition, the amine group can resonantly donate electrons from the nitrogen lone pair into the conjugated $\pi$-system, while the carboxylic acid or ester group has the ability to resonantly accept $\pi$-electron density due to the presence of the $\pi$-conjugated $\mathrm{sp}^{2}$ orbital in the carbonyl group. Since this resonance effect is only possible with the two groups being either in para or ortho positions, the basicities and possibly also the preferred protonation sites can vary significantly between the $p$-, $m$-, and $o$-isomers.

Currently, most investigations on protomers are carried out using gas-phase methods such as ion mobility spectrometry (IMS) and mass spectrometry (MS). For certain examples, the combination of both techniques was shown to be highly suitable for the separation of isobaric protonation isoforms. ${ }^{8,9,18-21}$ Protomers of molecules such as aniline, ${ }^{8}$ quinolone derivatives, ${ }^{9}$ meso-substituted porphyrins, ${ }^{8}$ benzocaines, ${ }^{20,21}$ melphalan, ${ }^{21}$ as well as $p$-aminobenzoic acid $^{18}$ have been distinguished. However, IMS only provides collision cross-section (CCS) values from which the location of the proton is difficult to deduce. To obtain additional information, experiments such as collision induced dissociation and hydrogen-deuterium exchange can be employed to tentatively assign the location of the proton. ${ }^{22,23}$

Since the protonation states of functional groups greatly influence their vibrational modes, infrared (IR) spectroscopic approaches were employed to determine the protonation sites of various molecular ions, including ABA derivatives. , 20,23-27 $^{2}$ Kass and coworkers measured the gas-phase IR spectrum of protonated $p$-ABA in the $2800-3600 \mathrm{~cm}^{-1}$ range. By analyzing characteristic $\mathrm{N}-\mathrm{H}$ and $\mathrm{O}-\mathrm{H}$ stretching vibrations they showed that for this molecule the preferred protonation site in vacuum is the carbonyl oxygen. ${ }^{23}$ Williams and coworkers used gasphase IR spectroscopy in the same wavelength range to show that the protonation sites in $p$-ABA and its methyl ester highly depend on the number of solvating water molecules. ${ }^{25}$ Most recently, two protomers of $p$-ABE (benzocaine) were successfully separated in the gas phase via IMS and their respective protonation sites unambiguously determined via gas-phase IR spectroscopy in the mid-IR range $\left(1000-2000 \mathrm{~cm}^{-1}\right) .{ }^{20}$

In this work, we employ a combination of IMS-MS with IR spectroscopy to investigate the $p$-, $m$-, and $o$-isomers of ABA and ABE. The experimental work is accompanied by ab initio calculations and the effects of substitution positions and of solvent molecules on protonation sites and on proton migration are discussed.

\section{Materials and methods}

\subsection{Sample preparation}

All solvents, aminobenzoic acids and the corresponding aminobenzoic acid ethyl esters were purchased from Sigma-Aldrich (Taufkirchen, Germany). Sample solutions were prepared by dissolving $\mathrm{ABA}$ or $\mathrm{ABE}$ at a concentration of $100 \mu \mathrm{M}$ in either water/methanol $(1 / 1, v / v)$ or in pure acetonitrile. An aliquot $(5-10 \mu \mathrm{L})$ of sample solution was then loaded into an in-houseprepared $\mathrm{Pd} / \mathrm{Pt}$-coated borosilicate capillary for nano-electrospray ionization.

\subsection{Ion mobility mass spectrometry}

The home-built drift tube-ion mobility mass spectrometer is described in detail elsewhere..$^{20,28-30}$ Ions are generated via nano-electrospray ionization with a spray voltage of $0.85 \mathrm{kV}$, collected in an RF ion funnel and pulsed into a $80 \mathrm{~cm}$-long drift tube. In the drift tube they travel under the influence of a weak electric field (10-15 $\mathrm{V} \mathrm{cm}^{-1}$ ) through helium or nitrogen buffer gas (2-5 mbar) for ion mobility separation. At the end of the drift region, a second $\mathrm{RF}$ ion funnel collects and transports IM-separated ions into the high vacuum region. A quadrupole mass filter is employed for $\mathrm{m} / \mathrm{z}$ separation and the ion current recorded as a function of arrival time (ATD) of $m / z$-selected ions. Collision cross-sections are determined from the measured drift times using the Mason-Schamp equation. ${ }^{31}$

\subsection{Ion mobility-selective infrared spectroscopy}

After ion mobility separation a narrow drift-time window of 100-150 $\mu$ s width is selected by electrostatic deflection of unwanted ions. Following $\mathrm{m} / \mathrm{z}$ selection, the ions are irradiated by a $7 \mu$ s pulse of infrared photons (pulse energies $\sim 10-40 \mathrm{~mJ}$ ) provided by the Fritz Haber Institute Free Electron Laser (FHI FEL). ${ }^{32}$ When the IR wavelength is resonant with an IR active mode of the molecule, the absorption of multiple photons, followed by dissociation, can occur. ${ }^{33}$ The resulting fragment ions are monitored using a time-of-flight mass analyzer. IR spectra are measured by scanning the IR wavenumber in $3 \mathrm{~cm}^{-1}$ steps in the range from $1000 \mathrm{~cm}^{-1}$ to $1900 \mathrm{~cm}^{-1}$, averaging 20 mass spectra for each step, and plotting fragmentation efficiency as a function of wavenumber.

\subsection{Computational methods}

Optimized geometries, relative energies, and harmonic vibrational frequencies of neutral and protonated aminobenzoic acids (ABAs) and their ethyl esters (ABEs), either in water or in vacuum, were calculated at the B3LYP level of theory with a def2TZVP basis set using the Gaussian 09 program. ${ }^{34}$ Vibrational frequencies were scaled by 0.98 and the calculated stick spectrum convoluted with a Gaussian function of $10 \mathrm{~cm}^{-1}$ width to obtain a theoretical IR spectrum for comparison with the experiment. All energies were corrected to $298 \mathrm{~K}$. Gas-phase basicities $\left(-\Delta G_{298 \text {,gas }}\right)$ were determined from the Gibbs free energy of the protonated molecule $\left([\mathrm{M}+\mathrm{H}]^{+}\right)$relative to the neutral (M) via eqn (1).

$$
\Delta G_{298, \text { gas }}=G_{298, \text { gas }}\left([\mathrm{M}+\mathrm{H}]^{+}\right)-G_{298, \text { gas }}(\mathrm{M})-G_{298, \text { gas }}\left(\mathrm{H}^{+}\right)
$$


The basicity in water $\left(-\Delta G_{298, \text { aq }}\right)$ was determined using eqn (2), where $\Delta G_{298 \text {,hyd }}$ denotes the Gibbs free energy of solvation in water.

$$
\begin{aligned}
\Delta G_{298, \text { aq }}= & \Delta G_{298, \text { gas }}+\Delta G_{298, \text { hyd }}\left([\mathrm{M}+\mathrm{H}]^{+}\right)-\Delta G_{298, \text { hyd }}(\mathrm{M}) \\
& -\Delta G_{298, \text { hyd }}\left(\mathrm{H}^{+}\right)
\end{aligned}
$$

The $\Delta G_{298, \text { hyd }}\left([\mathrm{M}+\mathrm{H}]^{+}\right)$and $\Delta G_{298, \text { hyd }}(\mathrm{M})$ values were determined using the implicit solvent polarizable continuum model $(\mathrm{PCM})^{35}$ as implemented in Gaussian 09. For $\Delta G_{298 \text {,hyd }}\left(\mathrm{H}^{+}\right)$, a previously determined value $\left(-1104 \mathrm{~kJ} \mathrm{~mol}^{-1}\right)^{36}$ was used, since PCM without explicit solvent molecules yields significant error in the solvation energy of the proton. ${ }^{37}$

\section{Results and discussion}

\subsection{Ion mobility experiments}

Fig. 2 shows arrival time distributions (ATDs) of protonated $p$-, $m$ - and $o$-ABA and -ABE using $\mathrm{He}$ and $\mathrm{N}_{2}$ as buffer gas. Samples were sprayed from either water/methanol (red lines) or acetonitrile (blue lines). The corresponding mass spectra are shown in Fig. S1 and S2 (see ESI $\dagger$ ). Most ATDs in Fig. 2 show more
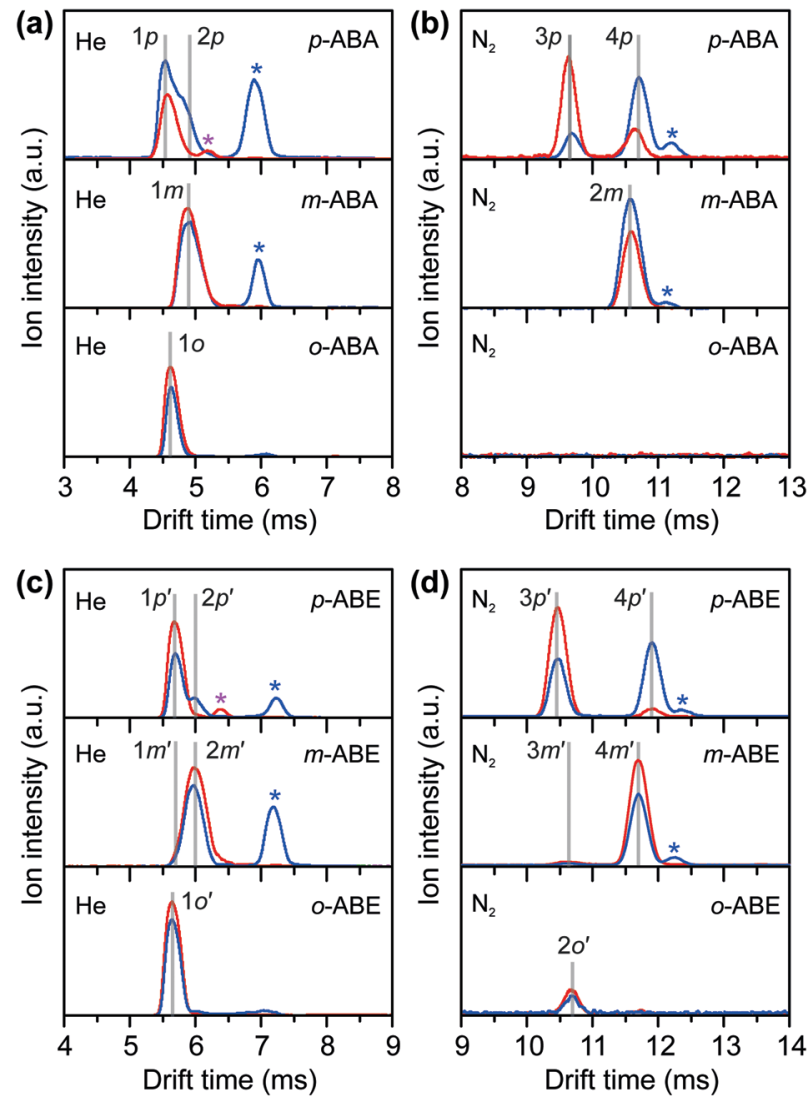

Fig. 2 Arrival time distributions (ATDs) of protonated para-, meta-, and ortho-aminobenzoic acid ( $a$ and $b$ ) and their corresponding ethyl esters (c and $d$ ) in $\mathrm{He}$ and $\mathrm{N}_{2}$ buffer gas. Red and blue lines represent ATDs obtained from samples sprayed from water/methanol and acetonitrile solution, respectively. Portions of ions in a $100 \mu$ s ATD windows, marked by gray lines, were transmitted for further investigation by IR spectroscopy. The peaks marked with an asterisk (*) are artifacts (see text). than one peak. Artifact peaks resulting from the dissociation of weakly bound solvent complexes after IM separation, but before $m / z$ selection, are marked by asterisks $\left(^{*}\right)$.

In the ATDs of protonated $p$-ABA sprayed from acetonitrile in He buffer gas, two partially resolved peaks, labeled $1 p$ and $2 p$, are observed. In contrast, in $\mathrm{N}_{2}$ buffer gas two well-separated features ( $3 p$ and $4 p$ ) are present. Peaks at similar positions are also found in the ATDs of protonated $p$-ABA sprayed from water/ methanol, with the difference that the high-mobility features $(\mathbf{1} \boldsymbol{p}$ and $3 p$ ) are more pronounced. This suggests that two distinguishable species with different ion mobilities are present and that their relative abundances depend on the solvent composition. Protonated $m$-ABA, on the other hand yields ATDs with only one peak independent of solvent composition and buffer gas selection (labeled $\mathbf{1 m}$ for $\mathrm{He}$ and $\mathbf{2 m}$ for $\mathrm{N}_{2}$ ). Protonated $o$-ABA shows only one sharp ATD peak (1o) in He. In the case of $\mathrm{N}_{2}$ drift buffer gas, no protonated $o$-ABA is observed, however an abundant fragment ion at $m / z=120$ is present, resulting from water loss. Presumably, protonated $o$-ABA is very fragile and collisions with $\mathrm{N}_{2}$ in the source region provide enough energy for efficient fragmentation.

The ATDs of protonated $p$-ABE show similar trends compared to $p$-ABA. As reported previously, ${ }^{20}$ two separable ATD features $\left(\mathbf{1} \boldsymbol{p}^{\prime}\right.$ and $\mathbf{2} \boldsymbol{p}^{\prime}$ in $\mathrm{He}$ as well as $\mathbf{3} \boldsymbol{p}^{\prime}$ and $\mathbf{4} \boldsymbol{p}^{\prime}$ in $\mathrm{N}_{2}$ ) are observed with their relative intensities strongly dependent on solvent composition. Protonated $m$-ABE shows predominantly one peak in the ATDs in both $\mathrm{He}$ and $\mathrm{N}_{2}$ buffer gas $\left(\mathbf{2 m ^ { \prime }}\right.$ and $\left.\mathbf{4} \boldsymbol{m}^{\prime}\right)$, however, a small amount of a high-mobility species $\left(\mathbf{1} \boldsymbol{m}^{\prime}\right.$ and $\mathbf{3} \boldsymbol{m}^{\prime}$ in He and $\mathrm{N}_{2}$, respectively) is observed as well. Protonated $o$-ABE also yields only one ATD peak $\left(\mathbf{1 o}^{\prime}\right.$ in $\mathrm{He}$ and $\mathbf{2} \boldsymbol{o}^{\prime}$ in $\left.\mathrm{N}_{2}\right)$, regardless of the solvent composition.

The corresponding collision cross-sections (CCSs) of the ATD peaks in Fig. 2 are summarized in Table 1. The differences in CCS between the high- and low-mobility features of the $p$ - or $m$-isomers are $4-8 \%$ in He and exceed $15 \%$ in $\mathrm{N}_{2}$ drift gas. Since very little conformational diversity is expected for either ABA or $\mathrm{ABE}$, those large differences in the CCS cannot be attributed to different conformers. Instead, the presence of different protomers is a more likely explanation. Indeed, several ion mobility studies have reported that protomers can have different ion mobilities, even though their shapes and structures are nearly identical..$^{8,9,18,20,21}$ In the case of $\mathrm{ABA}$ and $\mathrm{ABE}$, the proton can

Table 1 Experimental collision cross-sections (CCSs) of ion-mobility sepa-

\begin{tabular}{|c|c|c|c|c|}
\hline \multirow{2}{*}{$\frac{\mathrm{M}}{p-\mathrm{ABA}}$} & \multicolumn{2}{|c|}{$\mathrm{CCS}^{a}$ in $\mathrm{He}$} & \multicolumn{2}{|c|}{$\mathrm{CCS}^{a}$ in $\mathrm{N}_{2}$} \\
\hline & $1 p$ & 60 & $3 p$ & 129 \\
\hline & $2 p$ & 64 & $4 p$ & 143 \\
\hline$m$-ABA & $1 \mathrm{~m}$ & 63 & $2 m$ & 140 \\
\hline$o$-ABA & 10 & 59 & & \\
\hline \multirow[t]{2}{*}{$p$-ABE } & $1 p^{\prime}$ & 73 & $3 p^{\prime}$ & 136 \\
\hline & $2 p^{\prime}$ & 77 & $4 p^{\prime}$ & 155 \\
\hline \multirow{2}{*}{$m-\mathrm{ABE}$} & $1 m^{\prime}$ & 74 & $3 m^{\prime}$ & 136 \\
\hline & $2 m^{\prime}$ & 78 & $4 m^{\prime}$ & 153 \\
\hline$o-\mathrm{ABE}$ & $1 o^{\prime}$ & 72 & $2 o^{\prime}$ & 137 \\
\hline
\end{tabular}
rated protonated $A B A$ and $A B E$ isomers $\left([M+H]^{+}\right)$in $\mathrm{He}$ and $\mathrm{N}_{2}$ drift gas

${ }^{a}$ Unit in $\AA^{2}$. 
bind to either the amine ( $N$-protonation) or the carbonyl oxygen ( $O$-protonation). Since the vibrational frequencies of the amine and carbonyl groups depend on their protonation, significantly different IR spectra are expected for the $\mathrm{N}$ - and $\mathrm{O}$-protonated species.

\subsection{Gas-phase IR spectroscopy}

IR-spectra of the species arising from different ATD features are obtained by selecting the corresponding $\mathrm{m} / \mathrm{z}$ values and drift times followed by IRMPD spectroscopy. ${ }^{20,38}$ To do so, the selected ions are irradiated by infrared photons and the photofragmentation efficiency in the range $1000-1900 \mathrm{~cm}^{-1}$ is plotted as a function of wavenumber. The resulting IR spectra are shown in Fig. 3 together with the theoretical IR spectra of the lowest energy structures of the $\mathrm{N}$ - and $\mathrm{O}$-protonated species determined by theory. The optimized geometries are given in Fig. S3 and S4 (see ESI $\dagger$ ). The calculated spectra of $N$ - and $O$-protonated species are quite different and visual inspection allows a unique assignment of the experimental data.

For protonated $p$-ABA (Fig. 3a), the data clearly show that the low-mobility species ( $2 p$ and $4 p$ ) are $N$-protonated, whereas high-mobility species $(\mathbf{1} p$ and $\mathbf{3 p})$ are $O$-protonated. The $2 p$ and $4 \boldsymbol{p}$ species yield IR spectra dominated by four well-resolved bands at 1085, 1180,1460, and $1770 \mathrm{~cm}^{-1}$. These bands are well reproduced in the calculations for the $N$-protonated species and, based on normal mode analysis, we can assign the two strong bands at 1460 and $1770 \mathrm{~cm}^{-1}$ to the umbrella modes of
$\mathrm{NH}_{3}{ }^{+}$and the stretching vibration of free $\mathrm{C}=\mathrm{O}$, respectively. Those two bands are clearly diagnostic for $N$-protonation. The IR spectra of $1 \boldsymbol{p}$ and $3 \boldsymbol{p}$ on the other hand are significantly different and the strong IR bands at 1500 and $1580 \mathrm{~cm}^{-1}$ fit very well with the bands calculated for an $O$-protonated molecule.

The IR spectra of protonated $m$-ABA (Fig. $3 \mathrm{~b}$ ) clearly indicate $\mathrm{N}$-protonation and the absence of an $\mathrm{O}$-protonated species. The IR spectra of both $\mathbf{1 m}$ and $\mathbf{2 m}$ are dominated by two IR bands, which can be assigned to the $\mathrm{NH}_{3}{ }^{+}$umbrella mode at $1450 \mathrm{~cm}^{-1}$ and the free $\mathrm{C}=\mathrm{O}$ stretching mode at $1770 \mathrm{~cm}^{-1}$.

For protonated $o$-ABA (Fig. 3c), the IR spectrum consists of a strong band at $1695 \mathrm{~cm}^{-1}$, partially-resolved bands around 1300-1500 $\mathrm{cm}^{-1}$, and small bands at $\sim 1200$ and $\sim 1600 \mathrm{~cm}^{-1}$. The lack of a band for a free $\mathrm{C}=\mathrm{O}$ group around $1770 \mathrm{~cm}^{-1}$ might at first glance suggest $O$-protonation. However, the experimental IR spectrum is not in good agreement with the calculated IR spectrum of an exclusively $O$-protonated species (see Fig. 3c, red trace). Instead, it fits very well with the calculated spectrum for a molecule in which the proton is shared between the amine and the carbonyl oxygen (blue trace).

In Fig. 3(d-f), the IR spectra of the ABE species are shown and compared to the respective theoretical spectra. The general appearance and the presence of the diagnostic free $\mathrm{C}=\mathrm{O}$ stretching mode can be used to determine $\mathrm{N}$ - or $\mathrm{O}$-protonation. For protonated $p$-ABE (Fig. 3d), the measured IR spectra of lowmobility species $\left(\mathbf{2} \boldsymbol{p}^{\prime}\right.$ and $\left.\mathbf{4} \boldsymbol{p}^{\prime}\right)$ are in excellent agreement with (a) $p-A B A$



(d) $p-A B E$ (b) $m-\mathrm{ABA}$



in $\mathrm{N}_{2}$

$\mathrm{He}$

(c) o-ABA

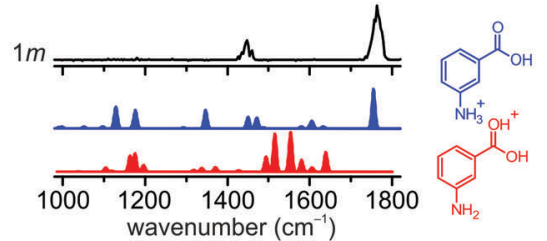

(e) $m$-ABE
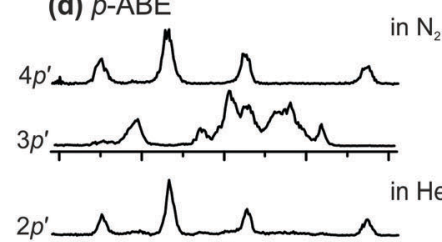

in $\mathrm{He}$



in $\mathrm{N}_{2}$
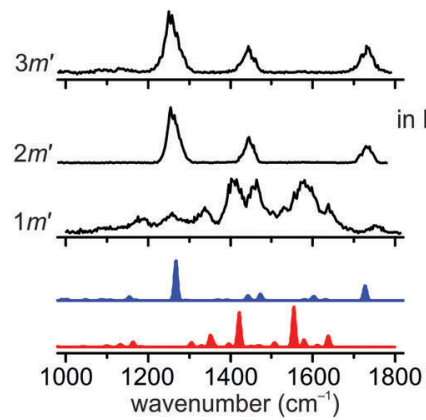

in $\mathrm{He}$

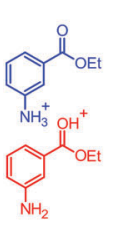

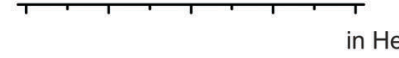



(f) $0-A B E$

in $\mathrm{N}_{2}$

Fig. 3 Gas-phase IR spectra of protonated, ion mobility-selected (a) $p$-, (b) $m$-, and (c) o-aminobenzoic acid, and protonated (d) $p$-, (e) $m$-, and (f) $O$-aminobenzoic acid ethyl ester. The red and blue traces represent the theoretical IR spectra of $O$ - and $N$-protonated species, respectively. All spectra were averages of two independent scans. 
the calculated IR spectrum for $N$-protonated $p$-ABE, showing a diagnostic IR feature at $1745 \mathrm{~cm}^{-1}$ that can be assigned as the stretching vibrations of free $\mathrm{C}=\mathrm{O}$. The high-mobility species $\left(1 p^{\prime}\right.$ and $\left.3 \boldsymbol{p}^{\prime}\right)$ on the other hand stem from $O$-protonated species, showing characteristic IR bands at 1410 and $1620 \mathrm{~cm}^{-1}$.

For protonated $m$-ABE (Fig. 3e), the dominant ATD features $\left(2 m^{\prime}\right.$ and $\left.4 m^{\prime}\right)$ originate from $N$-protonation. Three well-separated IR bands at 1280, 1450, and $1740 \mathrm{~cm}^{-1}$ fit very well with calculated IR bands for C-OEt stretching, $\mathrm{NH}_{3}{ }^{+}$umbrella, and free $\mathrm{C}=\mathrm{O}$ stretching modes, respectively. In contrast, the very low abundance high-mobility species $\left(\mathbf{1} m^{\prime}\right.$ and $\left.3 m^{\prime}\right)$ result from $O$-protonation. Despite the limited spectral quality and possible overlap with $\mathbf{2} \boldsymbol{m}^{\prime}$, the overall IR spectrum of $\mathbf{1} \boldsymbol{m}^{\prime}$ matches very well the calculated spectrum of $O$-protonated $m$-ABE.

For protonated $o$-ABE, the IR spectrum shows that the proton is shared by the carbonyl oxygen and the amine (Fig. 3f) as also observed for $o$-ABA.

In summary, the data show that the low-mobility (larger CCS) species in protonated $p$-ABE or -ABA are $N$-protonated, whereas high-mobility species (smaller CCS) are $O$-protonated. Protonated $m$-isomers are dominantly $N$-protonated, however for $m$-ABE, small amounts of $O$-protonated species are observed. Protonated $o$-isomers have a proton shared by the carbonyl oxygen and the amine ( $\mathrm{N}, \mathrm{O}$-protonated). The thermodynamic and kinetic influences that give rise to the large differences in protonation site of the $p$-, $m$-, and $o$-isomers will be discussed in the following sections.

\subsection{Thermodynamic stability}

To obtain further insights into the protonation of $\mathrm{ABA}$ and $\mathrm{ABE}$, the Gibbs free energies for both $N$ - and $O$-protonation at $298 \mathrm{~K}$ were calculated in vacuum (Fig. $4 \mathrm{a}$ and c) and in water solvent (Fig. 4b and d).

As shown in Fig. $4 \mathrm{a}$ and c, the $O$-protonated $p$-isomers are $37 \mathrm{~kJ} \mathrm{~mol}^{-1}(p-\mathrm{ABA})$ and $52 \mathrm{~kJ} \mathrm{~mol}^{-1}(p-\mathrm{ABE})$ more stable in the gas phase than the respective $N$-protonated species. For the $m$-isomers, however, the differences in stabilities between $O$ - and $N$-protonated gas phase isomers are within $6-7 \mathrm{~kJ} \mathrm{~mol}^{-1}$. In the case of the $O$-isomers, an $O$-protonated isomer could be calculated. Protonation at the amine group, on the other hand, always yielded structures in which the proton is shared between the carbonyl oxygen and amine ( $N, O$-protonated) which are significantly more stable than the exclusively $O$-protonated species.


nation of benzoic acid, benzoic acid ethyl ester, and aniline are shown in Fig. 4a and c. Intrinsically, the amine group in aniline is more basic than the carbonyl oxygen in benzoic acid or its ester. However, when both groups are present simultaneously, inductive and resonance effects which significantly contribute to the stabilities can occur. When the carbonyl oxygen in $p$-ABA and $p$-ABE becomes protonated, the amine group can donate


Fig. 4 Gibbs free energy at $298 \mathrm{~K}$ for protonation of $p-, m-$, and $o$-aminobenzoic acid (a and $\mathrm{b}$ ) and their corresponding ethyl esters (c and d) either in vacuum $\left(\Delta G_{298, \text { gas }}\right)$ or in water $\left(\Delta G_{298, a q}\right)$. For comparison, $\Delta G_{298, \text { gas }}$ and $\Delta G_{298, \text { aq }}$ values for benzoic acid, benzoic acid ethyl ester, and aniline are given. The $\varepsilon_{\mathrm{r}}$ denotes the relative permittivity. 
(a) para

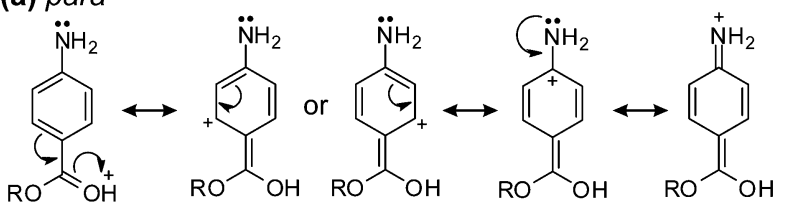

(b) meta



(c) ortho

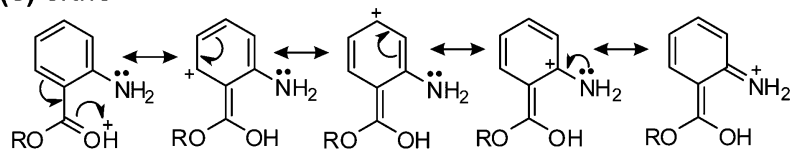

Fig. 5 Resonance stabilization of the $\mathrm{O}$-protonated (a) $p_{-}^{-}$, (b) $m-$, and (c) $0-A B A$ and $A B E$ isomers. The lone pair electrons of the amine in the $m$-isomer cannot participate in the resonance stabilization of the positive charge on the carbonyl oxygen.

electron density causing charge delocalization and resonant stabilization as schematically shown in Fig. 5a. In contrast, a positive charge at the amine ( $N$-protonation) is destabilized because the neutral carboxylic acid or ester group inductively withdraws electron density from the conjugated $\pi$-system and no resonance stabilization can take place. Compared to the gas phase basicity of aniline, benzoic acid and benzoic acid ethyl ester, these effects stabilize $O$-protonation by $50-53 \mathrm{~kJ} \mathrm{~mol}^{-1}$ for $p$-ABA and $p$-ABE, while $N$-protonation is destabilized by 20-30 kJ mol ${ }^{-1}$.

For the $m$-isomer, there is no resonance stabilization for $O$-protonation from the presence of the amine group, as both groups cannot be resonantly conjugated (see Fig. 5b). Some stabilization of $O$-protonation and destabilization of $N$-protonation via the inductive effect occurs $\left(20-25 \mathrm{~kJ} \mathrm{~mol}^{-1}\right.$ and 6-14 $\mathrm{kJ} \mathrm{mol}^{-1}$, respectively) much smaller than the corresponding values for the $p$-isomer.

In the case of $O$-protonated $o$-isomers the resonance effect can act similar to the $p$-isomers (see Fig. $5 \mathrm{c}$ ). As can be seen in Fig. 4a and c, however, this effect is rather small and most likely compensated for by stereoelectronic effects. Hence, the preferred structure for this molecule has the proton shared between the amine and carbonyl groups.

The known experimental gas-phase basicities of aniline and benzoic acid are 851 and $790 \mathrm{~kJ} \mathrm{~mol}^{-1}$, respectively. ${ }^{39}$ For aniline, the calculation here is in good agreement $\left(-3 \mathrm{~kJ} \mathrm{~mol}^{-1}\right)$, however for benzoic acid, theory overestimates $\Delta G_{298 \text {,gas }}$ by $17 \mathrm{~kJ} \mathrm{~mol}^{-1}$ (Fig. 4a). The reason may be the inherent bias of density functional theory towards charge delocalization, ${ }^{40,41}$ which would favor $O$-protonated species. However, it also appears that for the B3LYP calculations presented here this bias is small and does not affect the interpretations.

When the molecules are embedded in a dielectric medium such as water, the interaction of the medium with the charge becomes important. In Fig. $4 \mathrm{~b}$ and d, free energies are shown for the molecules in an environment with a relative permittivity of 78.4 (water). In the case of $N$-protonation the charge is highly localized compared to a small delocalization for $O$-protonation. This leads to a stronger interaction with the environment for $N$-protonation and a lower energy in all cases.

In the experiment exclusively $N$-protonated molecules are observed in all cases for which gas-phase $N$-protonation is predicted by theory. When gas-phase $O$-protonation is calculated to be lowest in energy, however, the experimental results consistently point to the presence of both species. The explanation for this observation is simple. In aqueous solution, $N$-protonation is always preferred. When $N$-protonation is also preferred in the gas phase, nothing changes. However, when the $O$-protonated variant is more stable in the gas phase, the proton has to migrate from its initial position, the amine, to the carbonyl oxygen. However, depending on the relative rates of proton migration and desolvation in the experiment, metastable, kinetically trapped $\mathrm{N}$-protonated species can remain. Such kinetic trapping and its solvent dependence are further investigated and discussed in the following sections.

\subsection{Acetonitrile complexes and proton-bound dimers}

The ratio between the $O$ - and $N$-protonated species of $p$-isomers in the ATDs strongly depends on the initial solvent composition. $N$-Protonated $p$-ABA and $p$-ABE are pronounced when spraying from acetonitrile, whereas $O$-protonated $p$-ABA and $p$-ABE are abundant for water/methanol mixtures. A possible origin of this observation might lie in the interactions of the charged site with solvent molecules. Protonated ABA-acetonitrile $(\mathrm{m} / \mathrm{z} 180)$ and ABE-acetonitrile ( $m / z 208)$ complexes are observed abundantly in the mass spectra taken when sprayed from acetonitrile. In contrast, water or methanol complexes of protonated ABA and $\mathrm{ABE}$ are largely absent (only observed at very low intensity when sprayed from water/methanol), which indicates that complexes with acetonitrile are considerably more stable than those with water or methanol (see Fig. S1 and S2 in ESI $\dagger$ ).

The IR spectrum of the protonated $p$-ABE-acetonitrile complex $\left([p-\mathrm{ABE}+\mathrm{ACN}+\mathrm{H}]^{+}, m / z 208\right)$ is shown in Fig. 6, top trace. Four strong IR bands at 1110, 1280, 1490, and $1755 \mathrm{~cm}^{-1}$ are observed. Comparison to calculated spectra for $N$ - and $\mathrm{O}$-protonated complexes indicates an $\mathrm{N}$-protonated complex. The IR bands at 1755 and $1280 \mathrm{~cm}^{-1}$ can be assigned to stretching vibrations of $\mathrm{C}=\mathrm{O}$ and $\mathrm{C}-\mathrm{OEt}$ in a free ester group. The IR band at $1490 \mathrm{~cm}^{-1}$ is likely to arise from the umbrella mode of $\mathrm{NH}_{3}{ }^{+}$interacting with acetonitrile. The results indicate that acetonitrile inhibits proton relocation from $\mathrm{N}$ - to $\mathrm{O}$-during desolvation, due to its strong interaction with the protonated amine. This interpretation is in agreement with previous studies in which kinetic trapping of the most stable solution protomer in the presence of acetonitrile has been suggested. ${ }^{22,23}$

This raises the question of how a proton can migrate from the amine to the carbonyl oxygen during the desolvation process. Proton relocation via intramolecular proton migration is unlikely because calculations predict high energy barriers for this process $\left(>200 \mathrm{~kJ} \mathrm{~mol}^{-1}\right)$, both in a dielectric medium $\left(\varepsilon_{\mathrm{r}}=78.4\right)$ and in vacuum (see Fig. S6 in ESI $\dagger$ ). Water molecules 


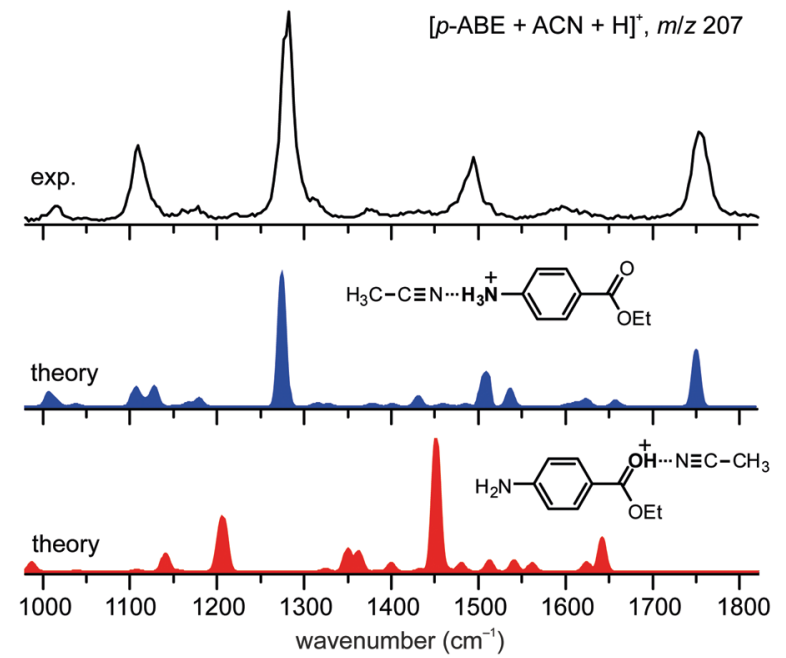

Fig. 6 Gas-phase IR spectra of $[p-\mathrm{ABE}+\mathrm{ACN}+\mathrm{H}]^{+}$complexes (top). Blue and red traces represent theoretical IR spectra of $\mathrm{N}$ - and $\mathrm{O}$-protonated species, respectively. The optimized geometries of acetonitrile complexes of $\mathrm{N}$ - and $\mathrm{O}$-protonated $p$-ABE are shown in Fig. S5 (see ESI $\dagger$ ).

can in principle assist proton transfer by forming a water bridge between the two protonation sites, as suggested by Williams and co-workers. $^{25}$ However, for the case of $p$-aminobenzoic acid methyl ester, calculations indicate that the formation of such a water bridge is energetically not favored. ${ }^{25}$ In addition, the presence of $O$-protonated $p$-ABA and $p$-ABE in aprotic acetonitrile cannot be explained by a solvent bridge model.

Alternatively, the formation of transient proton-bound dimers during desolvation could account for proton relocation.

As the solvent evaporates a proton-bound dimer can dissociate leading to two monomers where the proton will remain on the site having the highest basicity. Proton-bound dimers of $p$-ABA $\left([2 p-\mathrm{ABA}+\mathrm{H}]^{+}, m / z 275\right)$ and $\mathrm{ABE}\left([2 p-\mathrm{ABE}+\mathrm{H}]^{+}\right.$, $m / z$ 331) were observed in MS and IMS with He drift gas, and their IR spectra were recorded. The results are shown in Fig. 7 and compared to calculated IR spectra of several low energy geometries. The spectrum of $[2 p-\mathrm{ABA}+\mathrm{H}]^{+}$shows a strong IR band at $1180 \mathrm{~cm}^{-1}$ and several partially-resolved features in the $1300-1800 \mathrm{~cm}^{-1}$ range. The presence of an IR band at $1775 \mathrm{~cm}^{-1}$ is especially notable because it can be assigned to a stretching vibration of free $\mathrm{C}=\mathrm{O}$. Fig. 7c shows theoretical IR spectra of several low-energy proton-bound dimers. Proton bound $\mathrm{N}, \mathrm{N}$-dimers, in which a proton is shared between two amines, are found in all cases to be high in energy. The calculations indicate that there are two possibilities for the dimer between $N$-protonated and neutral $p$-ABAs: (1) the proton is shared between an amine and a carbonyl oxygen (N1,N,O-dimer), or (2) the proton is remote on the amine and the two carboxylic acids form the dimer bond (N2). Interestingly, the sum of theoretical IR spectra of $\mathbf{N} 1$ and $\mathbf{N} 2$ reproduces the experimental spectrum best. Calculated IR spectra for two very similar O,O-dimers (O1 and O2, in which a proton is located between two carbonyls) do not agree with the experimental results.

For $[2 p-\mathrm{ABE}+\mathrm{H}]^{+}$, neither the calculated spectra of the $\mathrm{N}, \mathrm{O}$-dimers ( $\mathbf{N 1}{ }^{\prime}$ and $\mathbf{N 2}{ }^{\prime}$ ) nor the $O, O$-dimers $\left(\mathbf{O 1}^{\prime}\right.$ and $\mathbf{O} 2^{\prime}$ ) fit the experimental spectrum well. However, the IR band at $1750 \mathrm{~cm}^{-1}$ indicates the presence of the stretching vibration of free $\mathrm{C}=\mathrm{O}$, which suggests the presence of $\mathrm{N}, \mathrm{O}$-dimers.

As shown in Table 2, N,O-dimers of $p$-ABA and $p$-ABE are energetically more favored in water than $O, O$-dimers, whereas $O, O$-dimers are the more stable in vacuum. Hence these $\mathrm{N}, \mathrm{O}$-dimers can survive most of the desolvation process during ESI $\dagger$ and then dissociate into a protonated monomer and a
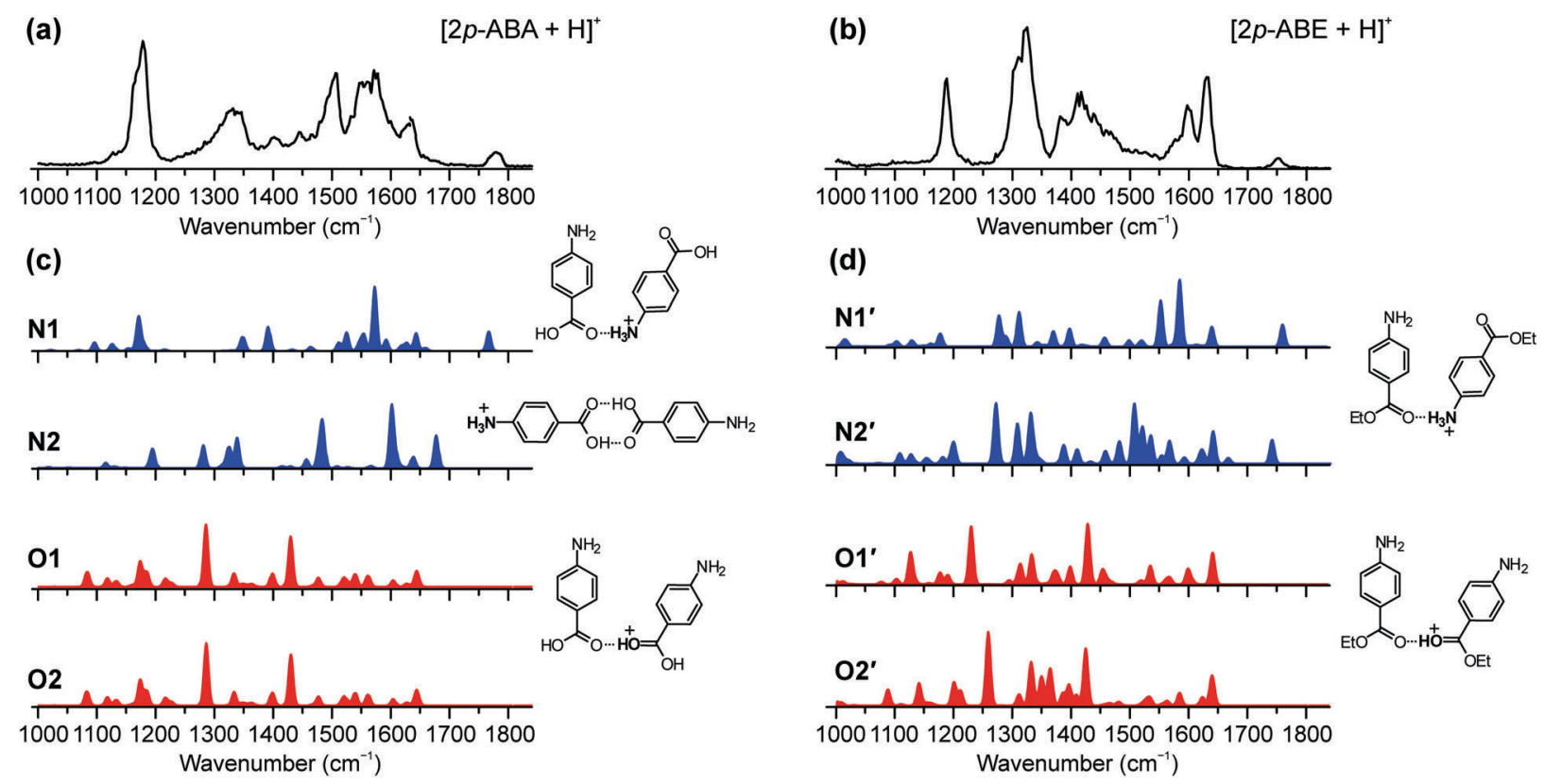

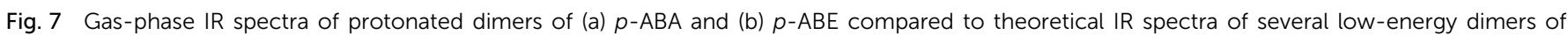
(c) $p-A B A$ and (d) $p$-ABE. The optimized 3-D geometries are given in Fig. S7 and S8 (see ESI $\dagger$ ). 
Table 2 Relative Gibbs free energy ${ }^{a}$ of the protonated dimers of $p$-ABA and $p$-ABE in Fig. 7

\begin{tabular}{|c|c|c|c|c|c|}
\hline & \multicolumn{2}{|c|}{$[2 p-\mathrm{ABA}+\mathrm{H}]^{+}$} & & \multicolumn{2}{|c|}{$[2 p-\mathrm{ABE}+\mathrm{H}]^{+}$} \\
\hline & In water & In vacuum & & In water & In vacuum \\
\hline N1 & 0.0 & 28.5 & N1 ${ }^{\prime}$ & 0.0 & 33.2 \\
\hline N2 & 4.1 & 64.0 & $\mathbf{N} 2^{\prime}$ & 0.4 & 32.7 \\
\hline 01 & 16.1 & 0.0 & O1' & 21.6 & 0.0 \\
\hline $\mathrm{O} 2$ & 16.6 & 0.3 & $\mathbf{O 2}^{\prime}$ & 22.0 & 0.4 \\
\hline
\end{tabular}

neutral molecule in vacuum, eventually leading to the observed $O$-protonated $p$-isomers.

\section{Conclusions}

Gas-phase IR spectroscopy was combined with ion mobility mass spectrometry to investigate the protonation sites for the protomers of $p$-, $m$-, and $o$-isomers of ABAs and ABEs. By identification of characteristic IR bands belonging to either protonated carbonyl or amine groups, the protonation site of each protomer was identified unambiguously. Each of the $p-, m-$, and $o$-isomers has a distinct protomeric population, with the $p$-isomers showing both $N$ - and $O$-protonated species, the $m$-isomers being predominantly $N$-protonated, and the $o$-isomers forming a structure in which the proton is shared between the amine and the carbonyl oxygen. Calculations indicate that the observed populations correlate with the stability of each protomer in the gas phase and further indicate the significance of resonance stabilization on the preferred protonation site. However, the observed protomer populations are not solely determined by the gas-phase stability. Kinetic trapping of $N$-protonated ions, which are the more stable species in solution, has a significant effect on the observed protomer population in the gas phase. In addition, for the $o$-isomer, proton sharing between the amine and the carbonyl oxygen is dominant.

Some of the species investigated here are known for their pharmacological activity in lipid bilayers. These bilayers exhibit a low relative permittivity $\left(\varepsilon_{\mathrm{r}}=2-4\right)$, which closely resembles that of the gas phase $\left(\varepsilon_{\mathrm{r}}=1\right)$. The observation of $O$-protonated species in the gas phase therefore implies that the protonation of ABA derivatives in biological membranes might be considerably different from that occurring in an aqueous medium. The fundamental findings of the present work, such as the protonation properties of the different isomeric structures as well as the proton relocation pathways, might therefore help to develop a better understanding of the biological activity of ABA derivatives and may be used for the design of novel drugs, which share the structural motifs of ABA.

\section{Acknowledgements}

M. T. B. gratefully acknowledges the support of the National Science Foundation (USA) for support under grant CHE-1301032 and support from the Alexander von Humboldt foundation.

\section{References}

1 K. A. Cox, S. J. Gaskell, M. Morris and A. Whiting, J. Am. Soc. Mass Spectrom., 1996, 7, 522-531.

2 J. Graton, M. Berthelot, J.-F. Gal, S. Girard, C. Laurence, J. Lebreton, J.-Y. Le Questel, P.-C. Maria and P. Nauš, J. Am. Chem. Soc., 2002, 124, 10552-10562.

3 D. Zagorevskii, M. Song, C. Breneman, Y. Yuan, T. Fuchs, K. Gates and C. M. Greenlief, J. Am. Soc. Mass Spectrom., 2003, 14, 881-892.

4 Z. Tozuka, H. Kaneko, T. Shiraga, Y. Mitani, A. Kawamura, A. Kagayama and A. Aoba, Drug Metab. Pharmacokinet., 2002, 17, 316-339.

5 O. Dopfer, N. Solcà, J. Lemaire, P. Maitre, M.-E. Crestoni and S. Fornarini, J. Phys. Chem. A, 2005, 109, 7881-7887.

6 Y.-P. Tu, J. Org. Chem., 2006, 71, 5482-5488.

7 L. Ashton, C. Johannessen and R. Goodacre, Anal. Chem., 2011, 83, 7978-7983.

8 P. M. Lalli, B. A. Iglesias, H. E. Toma, G. F. de Sa, R. J. Daroda, J. C. Silva Filho, J. E. Szulejko, K. Araki and M. N. Eberlin, J. Mass Spectrom., 2012, 47, 712-719.

9 C. Lapthorn, T. J. Dines, B. Z. Chowdhry, G. L. Perkins and F. S. Pullen, Rapid Commun. Mass Spectrom., 2013, 27, 2399-2410.

10 K. M. Hanson, E. Gratton and C. J. Bardeen, Free Radical Biol. Med., 2006, 41, 1205-1212.

11 M. E. Crisan, P. Bourosh, M. E. Maffei, A. Forni, S. Pieraccini, M. Sironi and Y. M. Chumakov, PLoS One, 2014, 9, e101892.

12 R. Michael, E. Richards and D. K. L. Xing, Int. J. Pharm., 1995, 116, 217-221.

13 R. M. E. Richards and D. K. L. Xing, J. Pharm. Biomed. Anal., 1994, 12, 1063-1068.

14 W. Schwarz, P. T. Palade and B. Hille, Biophys. J., 1977, 20, 343-368.

15 G. J. C. Basset, E. P. Quinlivan, S. Ravanel, F. Rébeillé, B. P. Nichols, K. Shinozaki, M. Seki, L. C. Adams-Phillips, J. J. Giovannoni, J. F. Gregory and A. D. Hanson, Proc. Natl. Acad. Sci. U. S. A., 2004, 101, 1496-1501.

16 A. Kluczyk, T. Popek, T. Kiyota, P. de Macedo, P. Stefanowicz, C. Lazar and Y. Konishi, Curr. Med. Chem., 2002, 9, 1871-1892. 17 C. Yanofsky, J. Biol. Chem., 1956, 223, 171-184.

18 J. L. Campbell, J. C. Y. Le Blanc and B. B. Schneider, Anal. Chem., 2012, 84, 7857-7864.

19 G. A. Bataglion, G. H. M. F. Souza, G. Heerdt, N. H. Morgon, J. D. L. Dutra, R. O. Freire, M. N. Eberlin and A. Tata, J. Mass Spectrom., 2015, 50, 336-343.

20 S. Warnke, J. Seo, J. Boschmans, F. Sobott, J. H. Scrivens, C. Bleiholder, M. T. Bowers, S. Gewinner, W. Schöllkopf, K. Pagel and G. von Helden, J. Am. Chem. Soc., 2015, 137, 4236-4242.

21 J. Boschmans, S. Jacobs, J. P. Williams, M. Palmer, K. Richardson, K. Giles, C. Lapthorn, W. A. Herrebout, F. Lemiere and F. Sobott, Analyst, 2016, 141, 4044-4054.

22 Z. Tian and S. R. Kass, Angew. Chem., Int. Ed., 2009, 48, 1321-1323. 
23 J. Schmidt, M. M. Meyer, I. Spector and S. R. Kass, J. Phys. Chem. A, 2011, 115, 7625-7632.

24 J. Oomens, A. R. Moehlig and T. H. Morton, J. Phys. Chem. Lett., 2010, 1, 2891-2897.

25 T. M. Chang, J. S. Prell, E. R. Warrick and E. R. Williams, J. Am. Chem. Soc., 2012, 134, 15805-15813.

26 A. Škríba, Š. Janková, J. Váňa, P. Barták, P. Bednář, P. Fryčák, L. Kučera, O. Kurka, K. Lemr, P. Macíková, E. Marková, P. Nováková, B. Papoušková, J. Skopalová, H. Švecová and J. Roithová, Int. J. Mass Spectrom., 2013, 337, 18-23.

27 J. Langer, A. Günther, S. Seidenbecher, G. Berden, J. Oomens and O. Dopfer, ChemPhysChem, 2014, 15, 2550-2562.

28 P. R. Kemper, N. F. Dupuis and M. T. Bowers, Int. J. Mass Spectrom., 2009, 287, 46-57.

29 S. Warnke, C. Baldauf, M. T. Bowers, K. Pagel and G. von Helden, J. Am. Chem. Soc., 2014, 136, 10308-10314.

30 S. Warnke, G. von Helden and K. Pagel, Proteomics, 2015, 15, 2804-2812.

31 E. A. Mason and E. W. McDaniel, Transport Properties of Ions in Gases, John Wiley \& Sons, 1988.

32 W. Schöllkopf, S. Gewinner, H. Junkes, A. Paarmann, G. von Helden, H. Bluem and A. M. M. Todd, Proc. SPIE, 2015, 9512, 95121L.

33 J. Oomens, B. G. Sartakov, G. Meijer and G. von Helden, Int. J. Mass Spectrom., 2006, 254, 1-19.

34 M. J. Frisch, G. W. Trucks, H. B. Schlegel, G. E. Scuseria, M. A. Robb, J. R. Cheeseman, G. Scalmani, V. Barone, B. Mennucci, G. A. Petersson, H. Nakatsuji, M. Caricato, X. Li, H. P. Hratchian, A. F. Izmaylov, J. Bloino, G. Zheng,
J. L. Sonnenberg, M. Hada, M. Ehara, K. Toyota, R. Fukuda, J. Hasegawa, M. Ishida, T. Nakajima, Y. Honda, O. Kitao, H. Nakai, T. Vreven, J. A. Montgomery Jr., J. E. Peralta, F. Ogliaro, M. J. Bearpark, J. Heyd, E. N. Brothers, K. N. Kudin, V. N. Staroverov, R. Kobayashi, J. Normand, K. Raghavachari, A. P. Rendell, J. C. Burant, S. S. Iyengar, J. Tomasi, M. Cossi, N. Rega, N. J. Millam, M. Klene, J. E. Knox, J. B. Cross, V. Bakken, C. Adamo, J. Jaramillo, R. Gomperts, R. E. Stratmann, O. Yazyev, A. J. Austin, R. Cammi, C. Pomelli, J. W. Ochterski, R. L. Martin, K. Morokuma, V. G. Zakrzewski, G. A. Voth, P. Salvador, J. J. Dannenberg, S. Dapprich, A. D. Daniels, Ö. Farkas, J. B. Foresman, J. V. Ortiz, J. Cioslowski and D. J. Fox, Gaussian 09, Revision D.01, Wallingford, CT, USA, 2013.

35 J. Tomasi, B. Mennucci and R. Cammi, Chem. Rev., 2005, 105, 2999-3094.

36 M. D. Tissandier, K. A. Cowen, W. Y. Feng, E. Gundlach, M. H. Cohen, A. D. Earhart, J. V. Coe and T. R. Tuttle, J. Phys. Chem. A, 1998, 102, 7787-7794.

37 E. F. da Silva, H. F. Svendsen and K. M. Merz, J. Phys. Chem. A, 2009, 113, 6404-6409.

38 A. Masson, M. Z. Kamrath, M. A. S. Perez, M. S. Glover, U. Rothlisberger, D. E. Clemmer and T. R. Rizzo, J. Am. Soc. Mass Spectrom., 2015, 26, 1444-1454.

39 E. P. L. Hunter and S. G. Lias, J. Phys. Chem. Ref. Data, 1998, 27, 413-656.

40 A. J. Cohen, P. Mori-Sánchez and W. Yang, Science, 2008, 321, 792-794.

41 X. Cheng, Y. Zhang, E. Jonsson, H. Jonsson and P. M. Weber, Nat. Commun., 2016, 7, 11013. 